\title{
Double-clad fibers for high-power fiber lasers
}

\author{
Pavel Peterka \\ Institute of Photonics and Electronics, The Czech Academy of Sciences, Chaberska 57, 18251 Prague, Czechia
}

Fiber lasers are considered as one of the youngest and most rapidly developing branch of lasers. In fact, we may observe recurrent increased interest in fiber lasers in time driven by various motivations and societal conditions. Although fiber lasers were proposed in 1960, even before the first laser light flash and before the first single-mode optical fiber was drawn, the fiber laser devices became hot-topic of research only in mid-80's as the invention of erbium-doped fiber amplifiers revolutionized the world of telecommunications and fueled the global boom of the internet in 90's. A second revolution started just at the beginning of the third millennium. With the availability of high-power, high brightness diode lasers to pump rare-earth-doped double-clad (DC) fibers, it began the race for high power from Yb-doped single mode fiber lasers at wavelength at around $1 \mu \mathrm{m}[1,2]$. Major breakthroughs involve development of various kinds of components and fiber design for efficient coupling and combination of the optical pump and laser signal into the DC active fiber, microstructure and air-clad designs of the DC fiber and namely the invention of large mode area (LMA) fibers together with methods to maintain single mode operation of LMA fibers.

The high-power operation of fiber lasers was enabled by the invention of cladding pumping within a DC fiber structure. Such a fiber serves as an efficient transformer of the low-brightness, high-power radiation of the laser diodes (coupled into the large area inner cladding of the DC fiber) into a high-brightness, highpower laser beam coming out from the rare-earth-doped, narrow fiber core. Since the most common circular shape of optical fibers provides poor effective absorption of the pump, various cross-sectional shapes of DC fibers have been investigated both experimentally and theoretically in order to enhance the absorption of the multimode-pump, see examples in Fig. 1(a) [3-5]. The beneficial effect of mode mixing of the pump radiation by unconventional coiling was also observed, see example of the twisting effect of the two-fiber bundle in Fig. 1(b) [3].

(a)

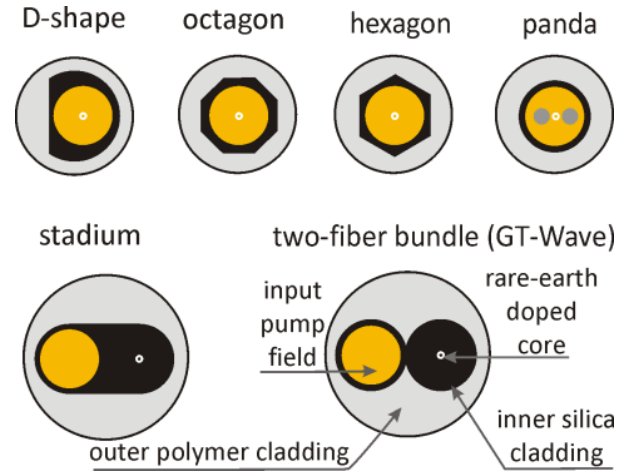

(b)

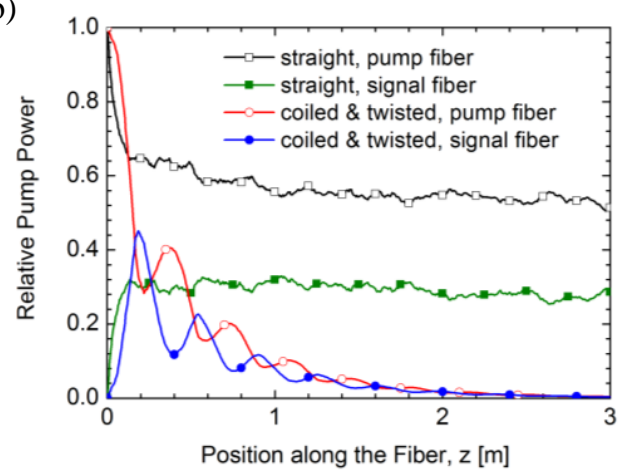

Fig. 1 (a) Examples of double-clad fiber cross sections of the inner cladding with broken circular symmetry for enhancement of pump absorption; yellow part schematically represent possible input pump distribution. (b) Numerical simulation of pump power distribution between the two fibers in the GT-Wave two-fiber bundle for the case of straight arrangement and coiled and twisted fiber bundle. Used with permission from ref. [3] (C[2016] IEEE).

This tutorial lecture will review principles of efficient pump absorption in double-clad fibers, fiber fabrication and characterization, pump and signal combiners, large mode area fibers, as well as practical limitations, including transversal [6] and longitudinal [7] mode instabilities, and emerging technologies.

\section{References}

[1] M. N. Zervas and C. A. Codemard, "High power fiber lasers: A review," IEEE J. Sel. Topics Quantum Electron. 20, 0904123 (2014).

[2] L. Dong and B. Samson, Fiber Lasers: Basics, Technology and Applications, (CRC Press, Taylor \& Francis Group, Boca Raton, 2017).

[3] P. Koska, P. Peterka, and V. Doya, "Numerical modeling of pump absorption in coiled and twisted double-clad fibers," IEEE J. Sel. Topics in Quantum Electron. 22, 4401508, (2016).

[4] P. Koska and P. Peterka, "Numerical analysis of pump propagation and absorption in specially tailored double-clad rare-earth doped fiber," Opt. Quantum Electron. 47, 3181 (2015).

[5] P. Koska, P. Peterka, J. Aubrecht, O. Podrazky, F. Todorov, M. Becker, Y. Baravets, P. Honzatko, and I. Kasik, "Enhanced pump absorption efficiency in coiled and twisted double-clad thulium-doped fibers," Opt. Express 24, 102 (2016).

[6] C. Jauregui, J. Limpert, and A. Tünnermann, "High-power fibre lasers," Nature Photonics 7, 861 (2013).

[7] P. Peterka, P. Koška, and J. Čtyroký, "Reflectivity of superimposed Bragg gratings induced by longitudinal mode instabilities in fiber lasers," IEEE J. Sel. Topics Quantum Electron. 24, 0902608 (2018). 